\title{
NONORTHOGONAL STAGNATION FLOW ON THE SURFACE OF A QUIESCENT FLUID-AN EXACT SOLUTION OF THE NAVIER-STOKES EQUATION
}

\author{
BY \\ TIANSHU LIU \\ University of Houston, Houston, Texas
}

\begin{abstract}
An exact solution of the Navier-Stokes equation that describes the twodimensional impingement of a light fluid on the surface of a heavier fluid at an arbitrary angle of incidence, assuming no surface distortion is obtained.
\end{abstract}

1. Introduction. The stagnation flow is one of the rare basic flows that can be described by exact solutions of the Navier-Stokes equation. Because of the great difficulties in generally solving nonlinear partial differential equations, exact solutions of the N-S equation are considerably restricted to those with a certain group invariance, i.e., similarity solutions. Hiemenz [1] first gave the similarity solution for the two-dimensional orthogonal stagnation flow on a rigid plate. The numerical work was improved later by Howarth [2]. The two-dimensional nonorthogonal stagnation flow on a solid plate was first solved by Stuart [3] and rediscovered by Tamada [4] and Dorrepaal [5]. Also, Wang [6] studied stagnation flow on the surface of a quiescent fluid without surface distortion. This paper gives an exact solution of the $\mathrm{N}-\mathrm{S}$ equation that depicts the two-dimensional nonorthogonal stagnation flow on the surface of a quiescent fluid. The inviscid flow pattern for this flow, sketched in Fig. 1 , is two-dimensional. This flow pattern has practical value. Peregrine [7] used this inviscid stagnation solution to construct the streamline pattern of the separating flow field of the splash. This paper will give a complete viscous stagnation solution for this flow pattern.

Consider that a lighter fluid blows downward at an incident angle $\theta$ on a quiescent heavier fluid. In order to obtain an exact solution, we assume that the interface is free of any distortion. Two coordinate systems are used to describe respectively the flows of the lighter fluid and heavier fluid. The origins of the two coordinate systems are located at the intersection between the interface and the inviscid dividing streamline (Fig. 1). The relation between the two coordinate systems is

$$
\tilde{x}_{1}=\tilde{x}_{h}, \quad \tilde{y}_{1}=-\tilde{y}_{h},
$$

where the subscripts 1 and $h$ denote respectively the lighter fluid and heavier fluid.

Received July 16, 1990.

(C)1992 Brown University 


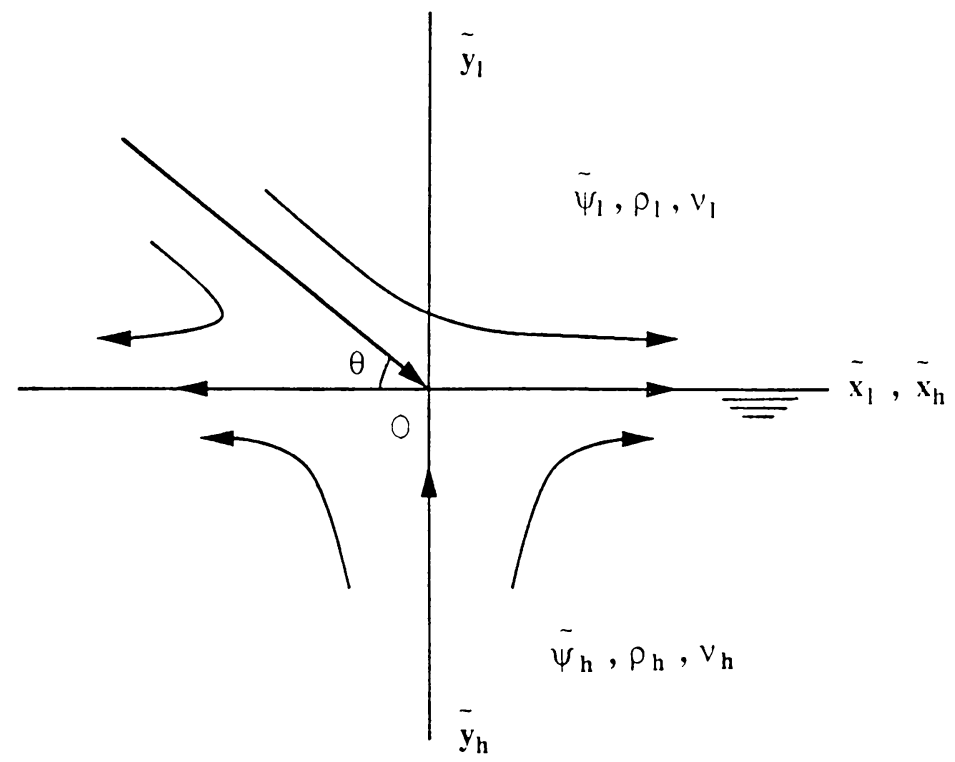

Fig. 1. Inviscid flow pattern.

The tilde $\sim$ denotes a dimensional quantity.

If the two flows are steady and the fluids are incompressible, then the twodimensional motions are governed by the vorticity equation

$$
\nu \nabla^{4} \tilde{\psi}=\left(\frac{\partial \tilde{\psi}}{\partial \tilde{y}} \frac{\partial}{\partial \tilde{x}}-\frac{\partial \tilde{\psi}}{\partial \tilde{x}} \frac{\partial}{\partial \tilde{y}}\right) \nabla^{2} \tilde{\psi},
$$

where $\tilde{\psi}$ is the streamfunction and $\nu$ is the kinematic viscosity. Here, $\tilde{\psi}$ can be decomposed into

$$
\tilde{\psi}=\tilde{\psi}_{1}\left(\tilde{y}_{1}>0\right) \text { or } \tilde{\psi}_{h}\left(\tilde{y}_{1}<0\right),
$$

where $\tilde{\psi}_{1}$ and $\tilde{\psi}_{h}$ are respectively the streamfunctions of the lighter fluid domain and heavier fluid domain. At the interface between the two domains, two matching conditions are needed to connect the two streamfunctions. At the interface, the velocity of the lighter fluid should be identical to that of the heavier fluid. Because of no surface distortion, the normal velocity components of both fluids vanish at the interface. Therefore, the velocity matching condition at the interface simply is

$$
\frac{\partial \tilde{\psi}_{1}}{\partial \tilde{y}_{1}}\left(\tilde{y}_{1}=0\right)=\frac{\partial \tilde{\psi}_{h}}{\partial \tilde{y}_{h}}\left(\tilde{y}_{h}=0\right) .
$$

Moreover, another condition is a viscous stress matching condition at the interface

$$
\rho_{1} \nu_{1} \frac{\partial^{2} \tilde{\psi}_{1}}{\partial \tilde{y}_{1}^{2}}\left(\tilde{y}_{1}=0\right)=-\rho_{h} \nu_{h} \frac{\partial^{2} \tilde{\psi}_{h}}{\partial \tilde{y}_{h}^{2}}\left(\tilde{y}_{h}=0\right) .
$$

The inviscid stagnation solution [5] is used as the far-field condition for the lighter fluid

$$
\tilde{\psi}_{1} \rightarrow \alpha_{1}\left[\tilde{y}_{1}^{2}(\cos \theta) / 2+\tilde{x}_{1} \tilde{y}_{1} \sin \theta\right] \quad\left(\tilde{y}_{1} \rightarrow \infty\right),
$$

where $\theta$ is the angle of incidence and $\alpha_{1}$ is a dimensional constant. 
For the heavier fluid, the far-field condition is

$$
\partial \tilde{\psi}_{h} / \partial \tilde{y}_{h} \rightarrow 0 \quad\left(\tilde{y}_{h} \rightarrow \infty\right) .
$$

2. Flow of the lighter fluid. By using the following nondimensional variables

$$
\psi_{1}=\tilde{\psi}_{1} / \nu_{1}, \quad y_{1}=\tilde{y}_{1}\left(\alpha_{1} / \nu_{1}\right)^{1 / 2}, \quad x_{1}=\tilde{x}_{1}\left(\alpha_{1} / \nu_{1}\right)^{1 / 2},
$$

equation (1.2) is normalized to

$$
\nabla^{4} \psi_{1}=\left(\frac{\partial \psi_{1}}{\partial y_{1}} \frac{\partial}{\partial x_{1}}-\frac{\partial \psi_{1}}{\partial x_{1}} \frac{\partial}{\partial y_{1}}\right) \nabla^{2} \psi_{1},
$$

where $\nu_{1}$ is the viscosity of the lighter fluid.

For the lighter fluid, a solution of Eq. (2.2) is constructed in the form of

$$
\psi_{1}=a^{-2}(\cos \theta) \int_{0}^{\eta} H(t) d t+a x_{1} F(\eta)
$$

where $\eta=a y_{1}$ and $a=(\sin \theta)^{1 / 2} . H(\eta)$ and $F(\eta)$ can be interpreted as the viscous modifications of the tangential and normal components of the inviscid solution (1.6). Substitution of expression (2.3) into Eq. (2.2) yields a fourth-order ordinary differential equation for $F(\eta)$ and a third-order ordinary differential equation for $H(\eta)$. Then, integrating the equation of $F(\eta)$ and using the far-field condition (1.6), one obtains

$$
\begin{gathered}
F^{\prime \prime \prime}+F F^{\prime \prime}-F^{2}+1=0 ; \\
F(0)=0, \quad F^{\prime}(0)=\beta_{2}, \quad F^{\prime}(\infty)=1,
\end{gathered}
$$

where the prime denotes differentiation with respect to $\eta$. The undetermined constant $\beta_{2}$ reflects the lateral motion of the interface. When $\beta_{2}=0$, the solution of Eq. (2.4a) is reduced to the Hiemenz solution. The asymptotic form of $F(\eta)$ in the far-field is written as

$$
F(\eta) \rightarrow \eta-A \quad(\eta \rightarrow \infty)
$$

where $A$ is obviously associated with $\beta_{2}$.

Solutions to (2.4a) at different $\beta_{2}$ were first found by Wang [6]. Equation (2.4a) is integrated numerically by using a shooting method. The dependences of $F^{\prime \prime}(0)$ and $A$ on $\beta_{2}$ are plotted in Fig. 2 (see p. 42). These results are identical with Wang's solution. For convenience, they can be quite accurately fitted by the polynomials

$$
F^{\prime \prime}(0)=1.2324-0.81597 \beta_{2}-0.47286 \beta_{2}^{2}+0.05657 \beta_{2}^{3}
$$

and

$$
A=0.6461-0.801 \beta_{2}+0.1922 \beta_{2}^{2}-0.03795 \beta_{2}^{3} .
$$

Similarly, by using the asymptotic condition (2.5), an equation for $H(\eta)$ is obtained

$$
\begin{aligned}
& H^{\prime \prime}+F H^{\prime}-F^{\prime} H+A=0 ; \\
& H(0)=\beta_{1}, \quad H^{\prime}(\infty)=1,
\end{aligned}
$$

where $\beta_{1}$ is a constant, which also reflects the motion of the interface. It will be shown later that both $\beta_{1}$ and $\beta_{2}$ are determined by the matching conditions. 


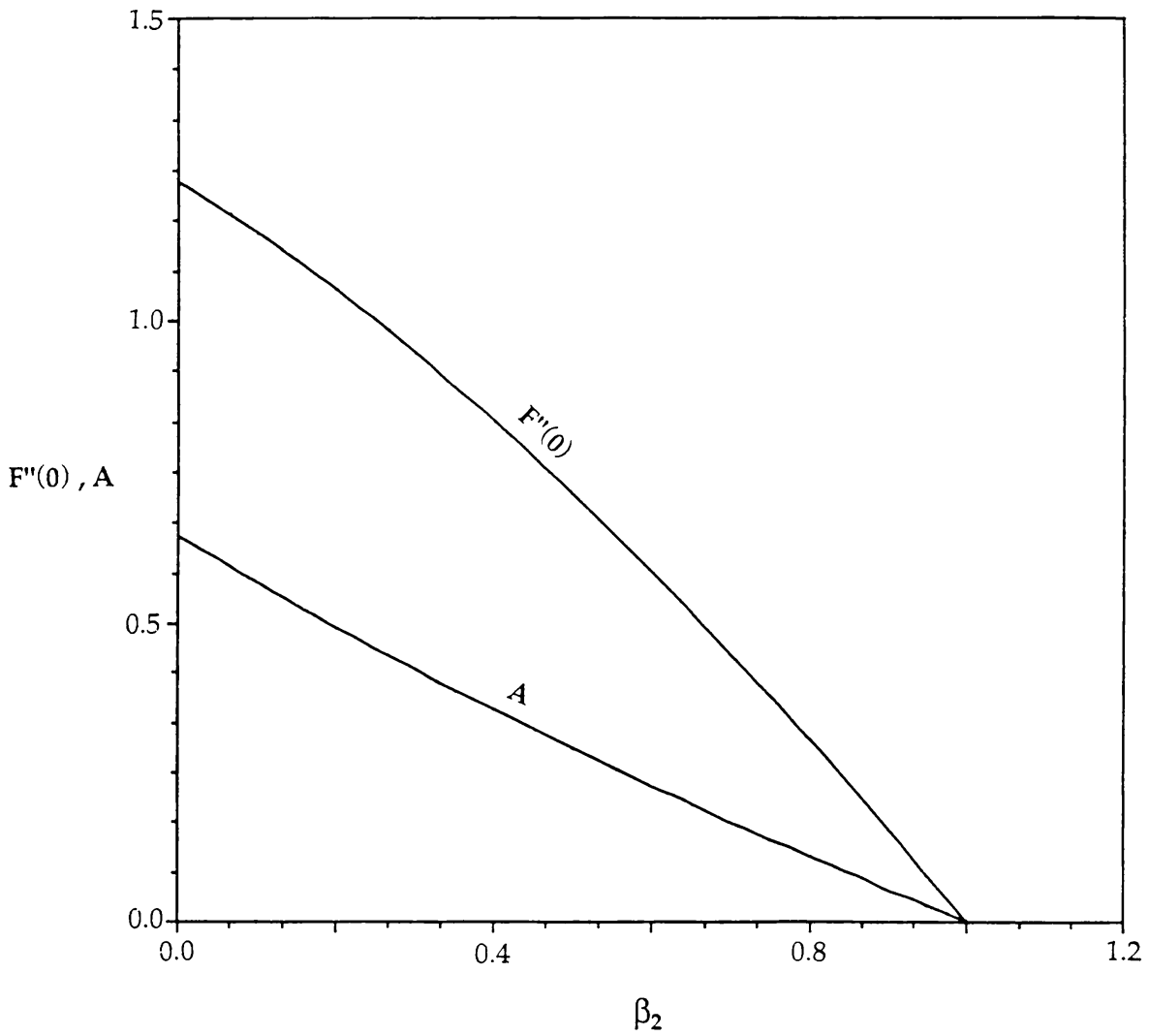

Fig. 2. Dependence of $F^{\prime \prime}(0)$ and $A$ on $\beta_{2}$.

By assuming that $H^{\prime}(0)$ is known, the boundary-value problem $(2.8 \mathrm{a})$ can be converted into an initial-value problem. Thus, a complete solution of Eq. (2.8a) can be formally given by

$$
H(\eta)=A F^{\prime}(\eta)+F^{\prime \prime}(\eta)\left\{c_{2}+c_{1} \int_{0}^{\eta} M(t) d t\right\},
$$

where

$$
\begin{gathered}
M(t)=F^{\prime \prime-2}(t) \exp \int_{0}^{t}-F(s) d s, \\
c_{1}=H^{\prime}(0) F^{\prime \prime}(0)-A F^{\prime \prime 2}(0)+\left(1-\beta_{2}^{2}\right)\left(\beta_{1}-A \beta_{2}\right), \\
c_{2}=F^{\prime \prime-1}(0)\left(\beta_{1}-A \beta_{2}\right) .
\end{gathered}
$$

The boundary value $H^{\prime}(0)$ depends on both $\beta_{1}$ and $\beta_{2}$. The numerical solution of Eq. (2.8a) shows that $H^{\prime}(0)$ is a linear function of $\beta_{1}$. The dependence of $H^{\prime}(0)$ on $\beta_{2}$ is shown in Fig. 3. The relation of $H^{\prime}(0)$ to $\beta_{1}$ and $\beta_{2}$ can be expressed by the following best-fitting formula

$$
H^{\prime}(0)=D_{1}\left(\beta_{2}\right) \beta_{1}+D_{2}\left(\beta_{2}\right)
$$




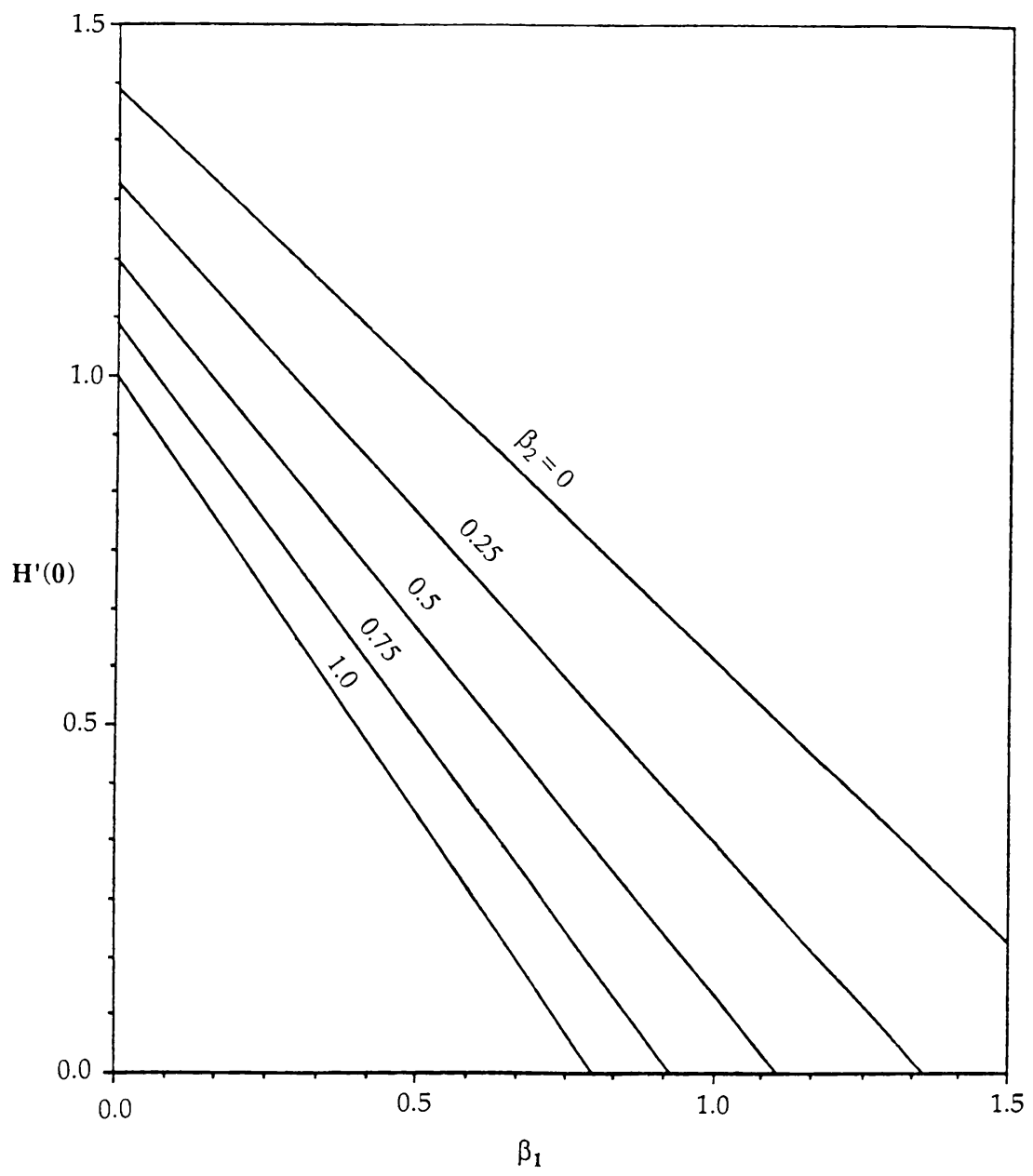

Fig. 3. Dependence of $H^{\prime}(0)$ on $\beta_{1}$ and $\beta_{2}$.

where

$$
\begin{gathered}
D_{1}\left(\beta_{2}\right)=-0.81235-0.5162 \beta_{2}+0.07622 \beta_{2}^{2}, \\
D_{2}\left(\beta_{2}\right)=1.4017-0.55276 \beta_{2}+0.15239 \beta_{2}^{2} .
\end{gathered}
$$

Thus, once $\beta_{1}$ and $\beta_{2}$ are known, $F(\eta)$ and $H(\eta)$ can be very easily solved as an initial-value problem, and the flow field of the lighter fluid is completely determined by expression (2.3).

3. Flow of the heavier fluid. Using the transformation

$$
\psi_{h}=\tilde{\psi}_{h} / \nu_{h}, \quad y_{h}=\tilde{y}_{h}\left(\alpha_{h} / \nu_{h}\right)^{1 / 2}, \quad x_{h}=\tilde{x}_{h}\left(\alpha_{h} / \nu_{h}\right)^{1 / 2},
$$

one obtains

$$
\nabla^{4} \psi_{h}=\left(\frac{\partial \psi_{h}}{\partial y_{h}} \frac{\partial}{\partial x_{h}}-\frac{\partial \psi_{h}}{\partial x_{h}} \frac{\partial}{\partial y_{h}}\right) \nabla^{2} \psi_{h}
$$

where $\alpha_{h}$ is a dimensional constant and $\nu_{h}$ is the kinematic viscosity of the heavier fluid. 
The stagnation solution of Eq. (3.2) is expressible in the form of

$$
\psi_{h}=\int_{0}^{y_{h}} Y(t) d t+x_{h} W\left(y_{h}\right) .
$$

Substituting expression (3.3) into Eq. (3.2) and using the velocity matching condition (1.4), one has

$$
\begin{array}{ll}
W^{\prime \prime \prime}+W W^{\prime \prime}-W^{\prime 2}=0 ; & \\
W(0)=0, & W^{\prime}(0)=\left(\alpha_{1} / \alpha_{h}\right) \beta_{2} \sin \theta, \quad W^{\prime}(\infty)=0
\end{array}
$$

and

$$
\begin{gathered}
Y^{\prime \prime}+W Y^{\prime}-W^{\prime} Y=0 ; \\
Y(0)=\left(\frac{\nu_{1} \alpha_{1}}{\nu_{h} \alpha_{h}}\right)^{1 / 2} \beta_{1}(\sin \theta)^{-1 / 2} \cos \theta, \quad Y(\infty)=0,
\end{gathered}
$$

where the prime denotes differentiation with respect to $y_{h}$.

Solutions to (3.4a) and (3.5a) can be given in closed form

$$
W\left(y_{h}\right)=m^{1 / 2}\left[1-\exp \left(-m^{1 / 2} y_{h}\right)\right]
$$

and

$$
Y\left(y_{h}\right)=Y(0) W^{\prime \prime}\left(y_{h}\right) / W^{\prime \prime}(0),
$$

where $m=\left(\alpha_{1} / \alpha_{h}\right) \beta_{2} \sin \theta$.

It is noted that the closed form solution to a homogeneous Hiemenz type equation (3.4a) was found by Crane [8] in modelling the flow due to a two-dimensional stretching plate. Finally, the streamfunction $\psi_{h}$ is written as

$$
\psi_{h}=\left(\frac{\alpha_{h}}{\nu_{h}}\right)^{1 / 2} m^{1 / 2} \widetilde{X}_{1}\left[1-\exp \left(-m^{1 / 2} y_{h}\right)\right],
$$

where

$$
\tilde{X}_{1}=\left(\frac{\nu_{1}}{\alpha_{1}}\right)^{1 / 2}\left[x_{1}+\cos \theta(\sin \theta)^{-3 / 2} \beta_{1} / \beta_{2}\right] .
$$

4. Determination of $\beta_{1}$ and $\beta_{2}$. Substitution of expressions (2.3) and (3.8) into the viscous stress matching condition (1.5) yields an algebraic system of equations,

$$
\begin{gathered}
H^{\prime}(0)=K \beta_{1} \beta_{2}^{1 / 2}, \\
F^{\prime \prime}(0)=K \beta_{2}^{3 / 2},
\end{gathered}
$$

where $K=\left(\rho_{h} / \rho_{1}\right)\left(\nu_{h} / \nu_{1}\right)^{1 / 2}$.

From (4.1), $\beta_{1}$ and $\beta_{2}$ only depend on a single parameter $K$, which represents the physical properties of the two fluids. The dependences of $\beta_{1}$ and $\beta_{2}$ on $K$, shown in Fig. 4, are determined by solving (4.1a) and (4.1b). If the densities and kinematic viscosity coefficients of the two fluids are known, we can find the corresponding values of $\beta_{1}$ and $\beta_{2}$. Therefore, $\psi_{1}$ and $\psi_{h}$ are completely known. 


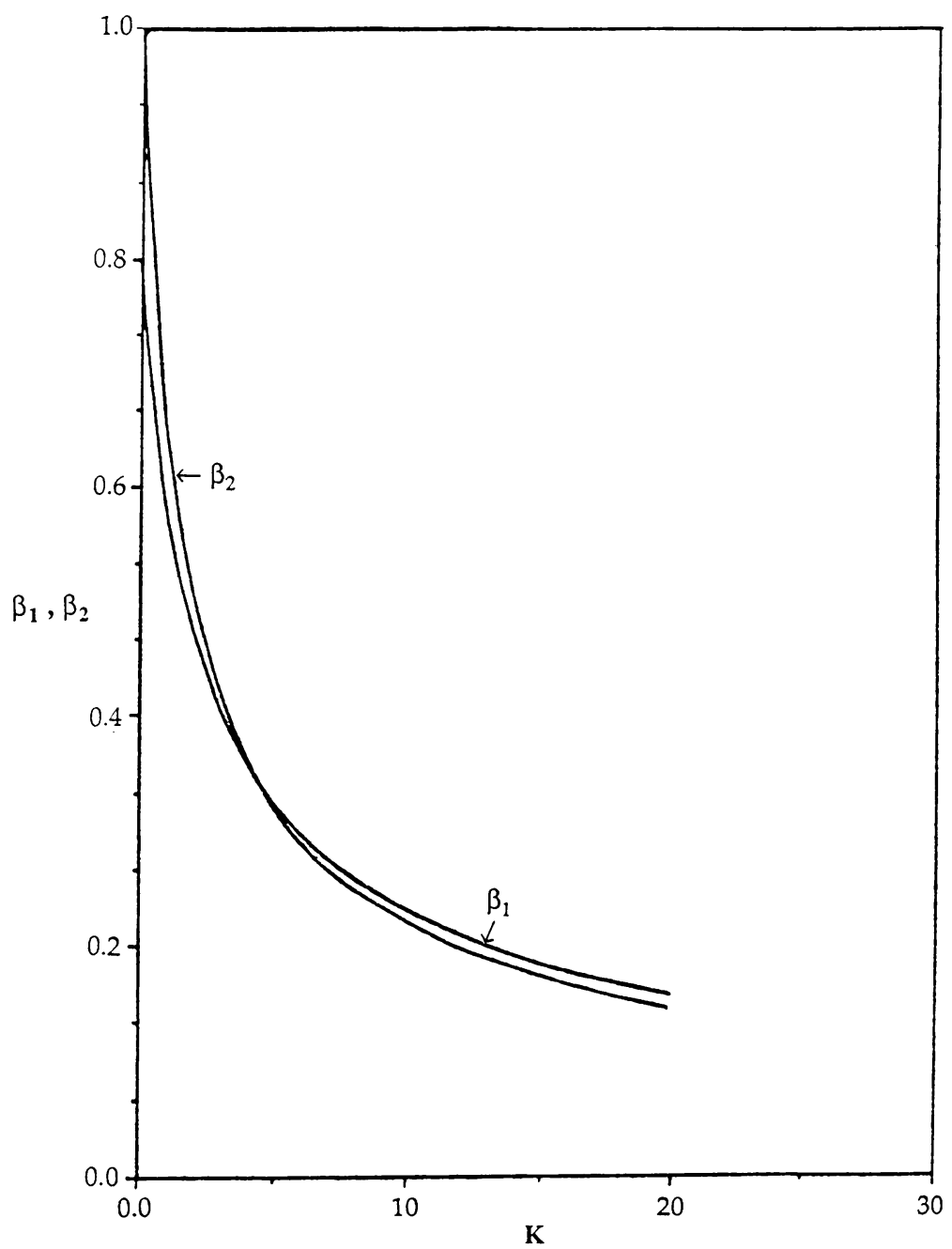

FIG. 4. Determination of $\beta_{1}$ and $\beta_{2}$.

5. Behavior of the flows near the interface. To understand the behavior of the flow of the lighter fluid near the interface, $F(\eta)$ and $H(\eta)$ are expanded in Taylor series at $\eta=0$

$$
\begin{gathered}
F(\eta)=\beta_{2} \eta+F^{\prime \prime}(0) \eta^{2} / 2+\left(\beta_{2}^{2}-1\right) \eta^{3} / 6+O\left(\eta^{4}\right), \\
H(\eta)=\beta_{1}+H^{\prime}(0) \eta+\left(\beta_{1} \beta_{2}-A\right) \eta^{2} / 2+O\left(\eta^{3}\right) .
\end{gathered}
$$

Substitution of $(5.1 \mathrm{a})$ and $(5.1 \mathrm{~b})$ into $(2.3)$ yields an asymptotic expression of the streamfunction $\psi_{1}$,

$$
\begin{array}{r}
\psi_{1}=a^{2} \beta_{2} X_{1} y_{1}\left(1+a^{2} y_{1}^{2} / 6\right)+\frac{1}{6}\left(\frac{D}{C}-A\right) a \cos \theta y_{1}^{2}\left\{y_{1}+3 C\left(\frac{D}{C}-A\right)^{-1} \tan \theta X_{1}\right. \\
\left.+a \tan \theta\left(\frac{D}{C}-A\right)^{-1} X_{1} y_{1}\right\}+O\left(y_{1}^{4}\right),
\end{array}
$$

where $X_{1}=x_{1}+\cos \theta(\sin \theta)^{-3 / 2}\left(\beta_{1} / \beta_{2}\right), C=F^{\prime \prime}(0)$, and $D=H^{\prime}(0)$. When $\beta_{2}=0,(5.2)$ is reduced to Dorrepaal's results [5]. 
The velocity of the lighter fluid near the interface is asymptotically

$$
u_{1}=\partial \tilde{\psi}_{1} / \partial \tilde{y}_{1}=\alpha_{1}(\sin \theta) \tilde{X}_{1}\left[F^{\prime}(0)+F^{\prime \prime}(0) \eta\right]+O\left(\eta^{2}\right)
$$

where $\tilde{X}_{1}=X_{1}\left(\nu_{1} / \alpha_{1}\right)^{1 / 2}$.

The velocity of the heavier fluid can be exactly expressed in a simple form,

$$
u_{h}=\partial \tilde{\psi}_{h} / \partial \tilde{y}_{h}=\alpha_{1}(\sin \theta) \beta_{2} \tilde{X}_{1} \exp \left(-m^{1 / 2} y_{h}\right) .
$$

From (5.3) and (5.4), one can find that the stagnation points of the two flows coincide at some position at $y_{1}=0$ or $y_{h}=0$ and

$$
\tilde{x}_{1}=\tilde{x}_{h}=-\left(\nu_{1} / \alpha_{1}\right)^{1 / 2}\left(\beta_{1} / \beta_{2}\right)(\sin \theta)^{-3 / 2} \cos \theta .
$$

The location of the stagnation point is dependent on the incident angle and the physical properties of the fluids. In contrast to the inviscid stagnation solution (1.4), the stagnation point in the exact solution deviates from the origin, except when $\theta=\pi / 2$, as shown in Fig. 5. This effect is obviously associated with viscosity. As $\theta$ goes to zero, the deviation of the stagnation point from the origin approaches infinity. When $\theta=\pi / 2$, the solution is reduced to the orthogonal stagnation flow solution.

The nondimensional dividing streamline $\psi_{1}=0$ of the lighter fluid near the interface can be expressed asymptotically by

$$
\begin{aligned}
a^{2} \beta_{2} X_{1} y_{1}\left(1+a^{2} y_{1}^{2} / 6\right)+\frac{1}{6}\left(\frac{D}{C}-A\right) a & \cos \theta y_{1}^{2}\left\{y_{1}+3 C\left(\frac{D}{C}-A\right)^{-1} \tan \theta X_{1}\right. \\
& \left.+a \tan \theta\left(\frac{D}{C}-A\right)^{-1} X_{1} y_{1}\right\}+O\left(y_{1}^{4}\right)=0 .
\end{aligned}
$$

When $\beta_{2}=0$, we obtain the slope of the dividing streamline at the stagnation point

$$
k_{s}=\frac{-3 C^{2}}{D-A C} \tan \theta
$$

which was given by Dorrepaal [5].

When $\beta_{2} \neq 0$, the first term in (5.6) cannot be neglected in the neighborhood of the stagnation point. Even when $\beta_{2}$ becomes smaller and smaller, we still cannot claim that the first term in (5.6) is less than the second term because $y_{1}$ also approaches zero at the neighborhood of the stagnation point. Hence, from (5.6), we directly derive the slope of the dividing streamline at $y_{1}=0$

$$
k_{s}=\frac{-2 a \beta_{2}}{C \cos \theta \tan \theta X_{1}} .
$$

Obviously, at the stagnation point $\left(X_{1}=0\right), k_{s}=\infty$ unless $\beta_{2}=0$.

Consequently, the slope of the dividing streamline of the lighter fluid at the stagnation point can be completely expressed by

$$
k_{s}= \begin{cases}x, & \beta_{2} \neq 0, \\ \frac{-3 C^{2}}{D-A C} \tan \theta, & \beta_{2}=0 .\end{cases}
$$




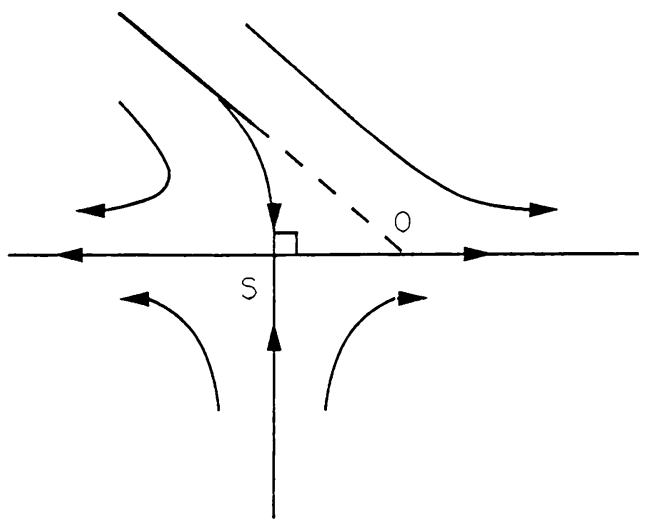

FIG. 5. Behavior of flows near the interface.

For a movable interface $\left(\beta_{2} \neq 0\right)$, the dividing streamline of the lighter fluid is always perpendicular to the interface (Fig. 5). If the interface is absolutely fixed $\left(\beta_{2}=0\right)$, Dorrepaal's results are recovered.

The vorticity distributions in the lighter fluid and heavier fluid domains are respectively

$$
\tilde{\omega}_{1}=\nabla^{2} \tilde{\psi}_{1}=\alpha_{1}\left(H^{\prime} \cos \theta+a^{3} x_{1} F^{\prime \prime}\right)
$$

and

$$
\tilde{\omega}_{h}=\nabla^{2} \tilde{\psi}_{h}=-\alpha_{h} m^{1 / 2}\left[Y(0)+m x_{h}\right] \exp \left(-m^{1 / 2} y_{h}\right) .
$$

Without loss of generality, the minus in (5.11) can be dropped. It is observed that the vorticity is discontinuous across the interface. From (5.10) and (5.11), a relation for vorticity discontinuity is

$$
\left.\frac{\tilde{\omega}_{h}}{\tilde{\omega}_{1}}\right|_{y_{1}=0}=\frac{\nu_{1} \rho_{1}}{\nu_{h} \rho_{h}}
$$

\section{REFERENCES}

[1] K. Hiemenz, Die Grenzschicht an einem in den gleichformigen Flussigkeitsstrom eingetauchten geraden Kreiszylinder, Dingl. Polytech. J. 326, 321 (1911)

[2] L. Howarth, On the calculation of the steady flow in the boundary layer near the surface of a cylinder in a stream, ARC RM 1632 (1935)

[3] J. T. Stuart, The viscous flow near a stagnation point when the external flow has uniform vorticity, J. Aerospace Sci. 26, 124-125 (1959)

[4] K. Tamada, Two-dimensional stagnation-point flow impinging obliquely on a plate wall, J. Phys. Soc. Japan 46, 310-311 (1979)

[5] J. M. Dorrepaal, An exact solution of the Navier-Stokes equation which describes non-orthogonal stagnation-point flow in two dimensions, J. Fluid. Mech. 163, 141-147 (1986)

[6] C. Y. Wang, Stagnation flow on the surface of a quiescent fluid-An exact solution of the NavierStokes equation, Quart. Appl. Math. 43, 215-223 (1985)

[7] D. H. Peregrine, The fascination of fluid mechanics, J. Fluid. Mech. 106, 50-80 (1981)

[8] L. J. Crane, Flow past a stretching plate, Z. Angew. Math. Phys. 21, 645-647 (1970) 